\title{
Correlative Imaging of Murine Pulmonary Valve Extracellular Matrix
}

\author{
Yifei Liu ${ }^{1}$, Yong-Ung Lee², Binbin Deng ${ }^{1}$, Christopher K. Breuer ${ }^{2}$, David W. McComb ${ }^{1}$ \\ ${ }^{1}$ Center for Electron Microscopy and Analysis, The Ohio State University, Columbus, OH, United \\ States \\ ${ }^{2}$ Nationwide Children’s Hospital, Department of Tissue Engineering, Columbus, OH, United States
}

Heart valves have purely biomechanical structures that respond to the fluid dynamic environment caused by heart palpitations. The pulmonary valve is responsible for unidirectional blood flow from the right ventricle of the heart to the pulmonary arteries, leading to the respiratory system. Healthy pulmonary valves consist of three leaflets, each compositionally and organizationally distinct [1]. Collagen is a fibrillar extracellular matrix (ECM) protein is that responsible for the mechanical strength of heart valves. Malformations and disorganization of collagen leads to biomechanical inefficiencies in the heart valve, which manifest as physiological conditions such as stenosis or regurgitation [2]. In order to comprehensively study the organization of the ECM, the physiological conditions of the valve must be controlled and related to the microstructure of the ECM. We are developing a correlative workflow in order to characterize the hierarchical organization of the collagen ECM and relate this to the biomechanical properties of the valve.

Black 6 mice (C57BL/6) were anesthetized with a solution of ketamine and xylazine, and were sacrificed according to IACUC approved protocols. An anastomosis was made between a pulmonary artery branch and a polyethylene catheter (0.025” OD). A fixation solution of $2.5 \%$ glutaraldehyde, $2 \%$ paraformaldehyde in $0.15 \mathrm{mM}$ cacodylate buffer was flowed from the arterial side at a pressure of 20 mmHg. The pulmonary valves were excised and inspected under and optical microscope (Leica Microsystems) to verify complete closure.

Specimens are stained with heavy metal atoms using a modified OTO procedure [3]. Tissues are stained with: $1.5 \%$ potassium ferrocyanide $(\mathrm{m} / \mathrm{v})$ and $2 \%$ osmium textroxide, $1 \%$ thiocarbohydrazide, $2 \%$ osmium textroxide (Electron Microscopy Sciences), 2\% uranyl acetate, and lead aspartate. Between each change of solution, tissues were washed with $0.15 \mathrm{mM}$ cacodylate buffer or double distilled water $\left(\mathrm{ddH}_{2} \mathrm{O}\right)$. Stained tissues dehydrated by ethanol treatments of 20\% 50\%, 70\%, 90\%, 100\%, followed by $100 \%$ acetone. Specimens were stepped through the graded mixtures of 25/75, 50/50, 75/25 and 100/0 (v/v) Durcupan ACM resin (Electron Microscopy Sciences) in acetone. Embedded tissues were cured at $60^{\circ} \mathrm{C}$ for 48 hours in Durcupan ACM resin.

Micro-computed tomography ( $\mu \mathrm{CT}$ ) was done using a Zeiss 520 Versa at the University of Manchester X-Ray Imaging Facility. Serial block face scanning electron microscopy (SBF-SEM) was done using FEI Teneo Volumescope in Eindhoven, Netherlands. Transmission electron microscopy (TEM) was conducted at the Center for Electron Microscopy and Analysis with FEI CM-200T TEM.

Figure 1A is an optical image of a representative closed heart valve after being excised from the heart. Figure $1 \mathrm{~B}$ shows a $\mu \mathrm{CT}$ volume rendering of a pulmonary valve in an open configuration without an applied transvalvular pressure. Using the $\mu \mathrm{CT}$ data, we can identify the position of the pulmonary valve and cut a specimen block oriented along the arterial axis. Figure $1 \mathrm{C}$ shows the volume rendering obtained from a series SBF-SEM images. A total of 100 slices were taken at a $20 \mathrm{~nm}$ slice thickness. At this 
resolution, it is possible to resolve fibrillar bundles. TEM specimens were made by ultramicrotome from the SBR-SEM specimen block. Directionality data obtained from TEM (Fig. 2A) complements the localized organizational information from SBF-SEM. The characteristic banding structure of collagen is seen by TEM, as shown in Figure 2B. The left arrow shows collagen fibrils that are parallel to the plane of the image and the right arrow shows fibrils oriented perpendicular to the plane of the image.

We have demonstrated the key steps to thoroughly characterizing the organization of the collagen extracellular matrix in a multiscale correlative workflow. Though we have tested each imaging system, we have yet to take an individual sample through the entire workflow. This procedure is currently being refined to distinguish differences between diseased and healthy valves as well as elucidating collagen maturation during heart valve development.

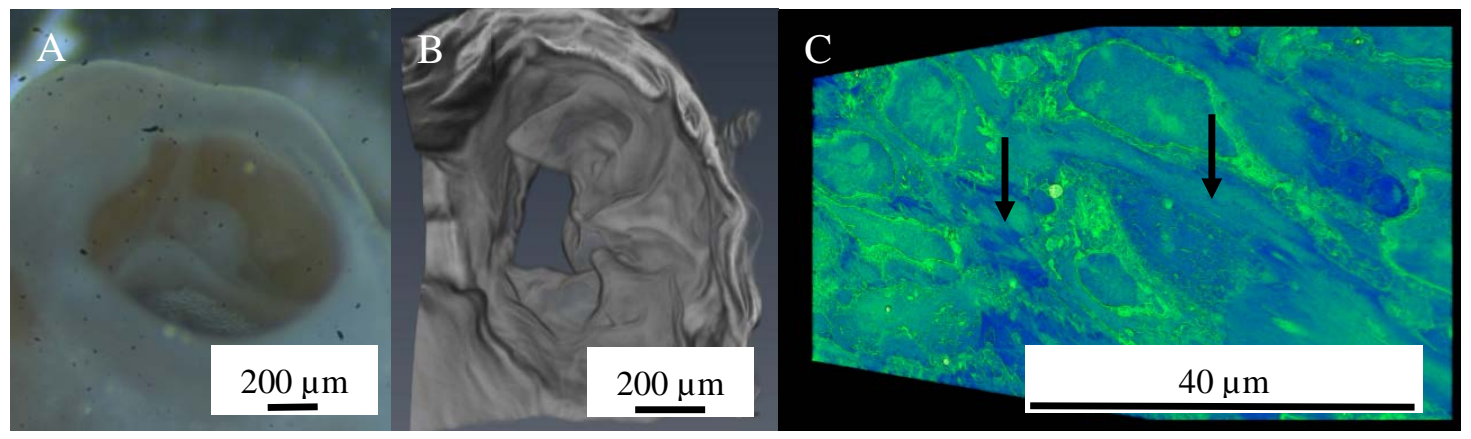

Figure 1. (A) Optical microscope capture of pulmonary valve in closed configuration. (B) Microcomputed tomography volume rendering of pulmonary valve. (C) 3-D rendering of pulmonary valve generated from serial SEM micrographs. Arrows indicate bundles of collagen.

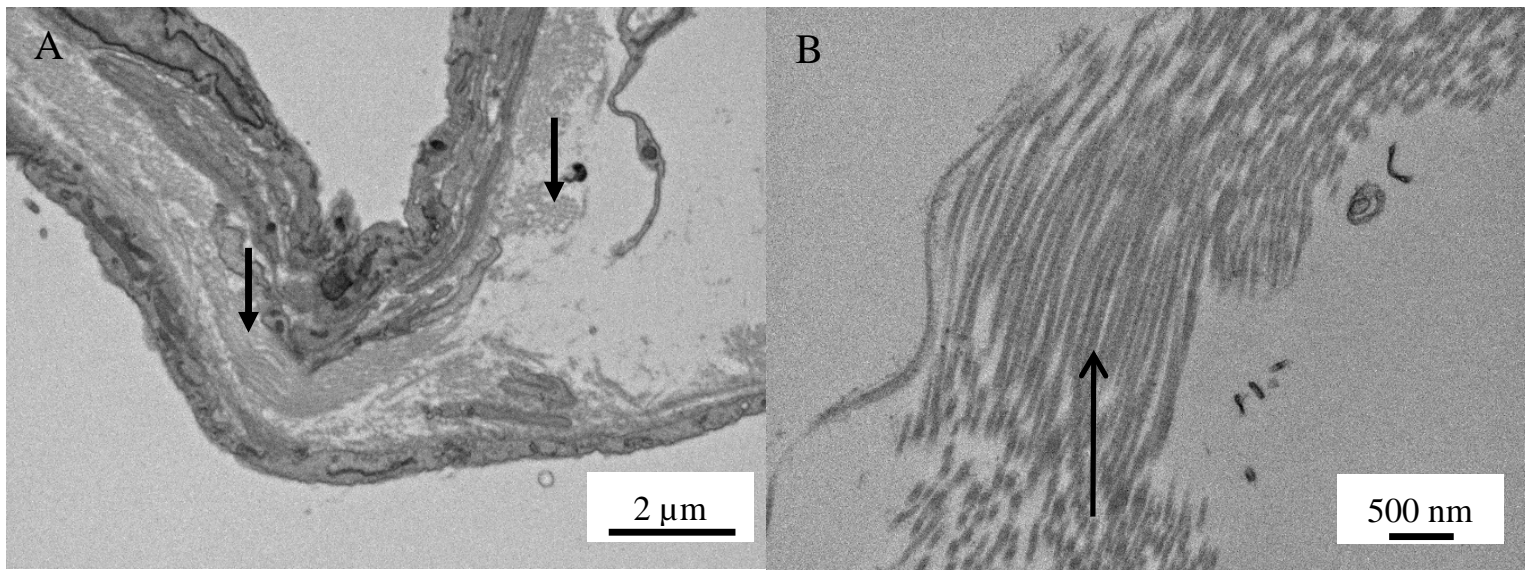

Figure 2. Transmission electron micrographs of pulmonary valve leaflet. Arrows correspond to collagen fibrils.

\section{References:}

[1] Gross L and MA Kugel, American Journal of Pathology 7.5 (1931), p. 0445-74

[2] Hinton RB and KE Yutzey, Annual Review of Physiology, Vol 73 (2011), p. 29-46

[3] Seliman AM, HL Wasserkrug and JS Hanker, Journal of Cell Biology 30.2 (1966), p. 424,+. 